\title{
The onset of ulcerative colitis
} upon Helicobacter pylori eradication in a 72-year-old woman: report of a rare case
with a 3-year follow-up

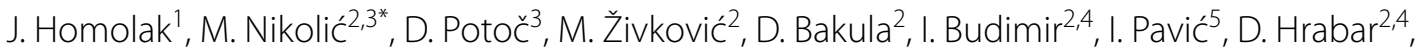
N. Ljubičićc ${ }^{2,3,4}$ and D. Vražićc

\begin{abstract}
Background: Epidemiological studies suggest an inverse association between $\mathrm{H}$. pylori infection/exposure and inflammatory bowel disease prevalence/incidence, however, there are no reports of individual patients who developed a "non-transient" ulcerative colitis (UC) following H. pylori eradication.

Case presentation: We report a case of a 72-year-old female with an elderly-onset UC developed upon H. pylori eradication and a 3-year follow-up of the progression to steroid-dependent colitis complicated with enteropathic arthritis and final containment of the disease with golimumab. In our patient, H. pylori eradication was associated with the development of pancolitis that evolved into clinically, endoscopically, and pathohistologically confirmed UC.

Conclusions: The case of our patient provides a unique clinical context for a growing body of literature suggesting molecular mechanisms involved in the interaction of genes, environment, and microbiota to be of critical importance in the etiopathogenesis of UC, and thus, provides a valuable set of complementary translational information for preclinical and epidemiological research on the topic.
\end{abstract}

Keywords: Case report, Helicobacter pylori, Ulcerative colitis, Inflammatory bowel disease, Golimumab

\section{Background}

Helicobacter pylori (H. pylori) is an important human pathogen with $50-90 \%$ prevalence depending on the stage of economic development [1]. H. pylori causes active gastritis associated with peptic ulcers, atrophic gastritis, gastric adenocarcinoma, and MALT lymphoma [2]. Furthermore, it is associated with unexplained iron deficiency anemia, idiopathic thrombocytopenic purpura, vitamin B12 deficiency and there is some early evidence for atherosclerosis, stroke, Alzheimer's and

\footnotetext{
*Correspondence: marko.nikolic72@gmail.com

${ }^{2}$ Gastroenterology and Hepatology Unit, University Hospital Centre

"Sestre Milosrdnice", Vinogradska 29, 10000 Zagreb, Croatia
}

Full list of author information is available at the end of the article
Parkinson's disease [2]. Increasing understanding of the serious pathophysiological consequences of $H$. pylori infection led to a general recommendation published in the Kyoto global consensus report that even individuals without clinically manifest disease should undergo eradication [3]. Interestingly, a growing body of research also reported a negative association of $H$. pylori and some diseases such as asthma [4] and inflammatory bowel disease (IBD) [5], however, the causality of these associations, their clinical relevance, and mechanisms of action are yet to be elucidated.

IBD is an umbrella term representing a group of chronic inflammatory diseases of the gastrointestinal tract. Ulcerative colitis (UC) and Crohn's disease (CD) 
are the most important clinical entities of IBD characterized by episodes of mucosal inflammation accompanied by abdominal pain, diarrhea, bloody stools, and weight loss [6]. In UC, the disease usually affects the rectum and the part of the colon, but in severe cases, the entire colon can be inflamed. Pathohistological findings usually reveal inflammation limited to the mucosa and submucosa with cryptitis and crypt abscesses [6], and molecular studies indicate increased TH1 and/or TH17 responses accompanied by enhanced production of tumor necrosis factor $\alpha$, interleukin 1- $\beta$, interferon $\gamma$, interleukin-17, interleukin-6, and IL-23 [7]. Diagnosis of IBD is usually done by colonoscopy combined with pathohistological examination of colonic biopsies, however exciting new diagnostic approaches such as virtual computed tomography (CT) colonoscopy (CT colonography) [8] and diffusionweighted imaging techniques [9] have also been proposed recently. Etiopathogenesis of UC is still unknown and recent evidence suggests pathophysiological processes arise as a consequence of a complex interaction of genetic predisposition, dysbiosis, environmental and immunological factors [6].

An inverse association between H. pylori infection/ exposure and prevalence/incidence of IBD has been reported by many epidemiological studies [5, 10-12]. A comprehensive meta-analysis on the prevalence of $H$. pylori infection in IBD (including UC subgroup) and non-IBD controls suggested a negative association after accounting for ethnicity, age, detection methods, and previous use of drugs (e.g. aminosalicylates and corticosteroids) [5]. Furthermore, an inverse association between $H$. pylori prevalence and disease severity was reported [13]. Several mechanisms have been proposed for the potential protective effect. It has been shown that $\mathrm{Hp}(2-20)$, an $H$. pylori ribosomal peptide reduces inflammation and upregulates mucosal damage repair in a rat model of colitis [14, 15]. Microbiota, an important player in IBD [16] is modulated by $H$. pylori [17]. H. pylori has been shown to reduce the gastric secretion of the proinflammatory satiety hormone leptin (increased in UC), and increase tolerogenic dendritic cells and suppressive regulatory T cells through IL-18 [5].

Although accumulating evidence suggests $H$. pylori might provide some beneficial anti-inflammatory effects, the results should be interpreted with caution. Observational retrospective studies are susceptible to bias, and appropriate adjustments are not always possible. The incidence of IBD is associated with economic development and "westernization" e.g. increased use of antibiotics, hygiene, cigarette smoking and consumption of processed foods, and decreased family size [18]. The aforementioned factors are also inversely associated with the $H$. pylori infection rates, so the observed association might "merely be a proxy for the hygiene hypothesis" [18].

Interestingly, in contrast to extensive epidemiological studies, case reports of patients developing IBD upon H. pylori eradication that would provide a "proof-ofconcept" are scarce. Jovanovic reported an onset of CD in a 28-year-old upon H. pylori eradication [19]. Tursi described a 34-year-old man and a 39-year-old woman who developed CD upon H. pylori eradication [20]. Nagami reported a patient requiring a subtotal colectomy following a relapse of $\mathrm{UC}$ associated with $H$. pylori eradication [21, 22], and Chiba reported a case of a 63-yearold developing a transient UC upon eradication therapy [22]. To the best of our knowledge no published reports describe the course of $H$. pylori eradication therapyinduced UC that required initiation of biological therapy to achieve satisfactory disease control.

We report a case of a 72-year-old woman who developed UC upon H. pylori eradication with a 3-year follow-up, and final containment of the disease following initiation of golimumab (Fig. 1).

\section{Case presentation}

A 72-year-old Caucasian female (height-161 cm; weight- $82 \mathrm{~kg} ;$ BMI- $31.6 \mathrm{~kg} / \mathrm{m}^{2}$ ) presented with a yearlong history of dyspeptic symptoms in 2017. The patient reported she was diagnosed with chronic gastritis and gastroesophageal reflux disease in 2016 (Fig. 2A). Past medical history included untreated osteoporosis diagnosed in 2005, thyroidectomy (toxic multinodular goiter in 2013 and a history of Hashimoto's thyroiditis), hypertension, and Reinke's edema-induced hoarseness. Family history and physical examination were unremarkable. The patient admitted to a 50-pack-year of cigarette smoking. She was regularly taking levothyroxine, rabeprazole, amlodipine, and reported penicillin allergy.

Esophagogastroduodenoscopy (EGD) and pathohistology were suggestive of moderate chronic active gastritis with incomplete intestinal metaplasia and $H$. pylori invasion in concordance with findings from 2016 (Fig. 2B). Eradication therapy with rabeprazole, clarithromycin, metronidazole, and a probiotic was initiated. The patient was encouraged to stop smoking.

Seven days in eradication, the patient reported to the emergency department due to redness and tongue swelling. The symptoms were resolved with intravenous methylprednisolone and intramuscular chloropyramine. Eradication was discontinued.

Two months later the patient underwent EGD with biopsy samples subjected to $H$. pylori antibiotic sensitivity testing. Penicillin allergy was ruled out with intracutaneous testing. A combination of esomeprazole, 


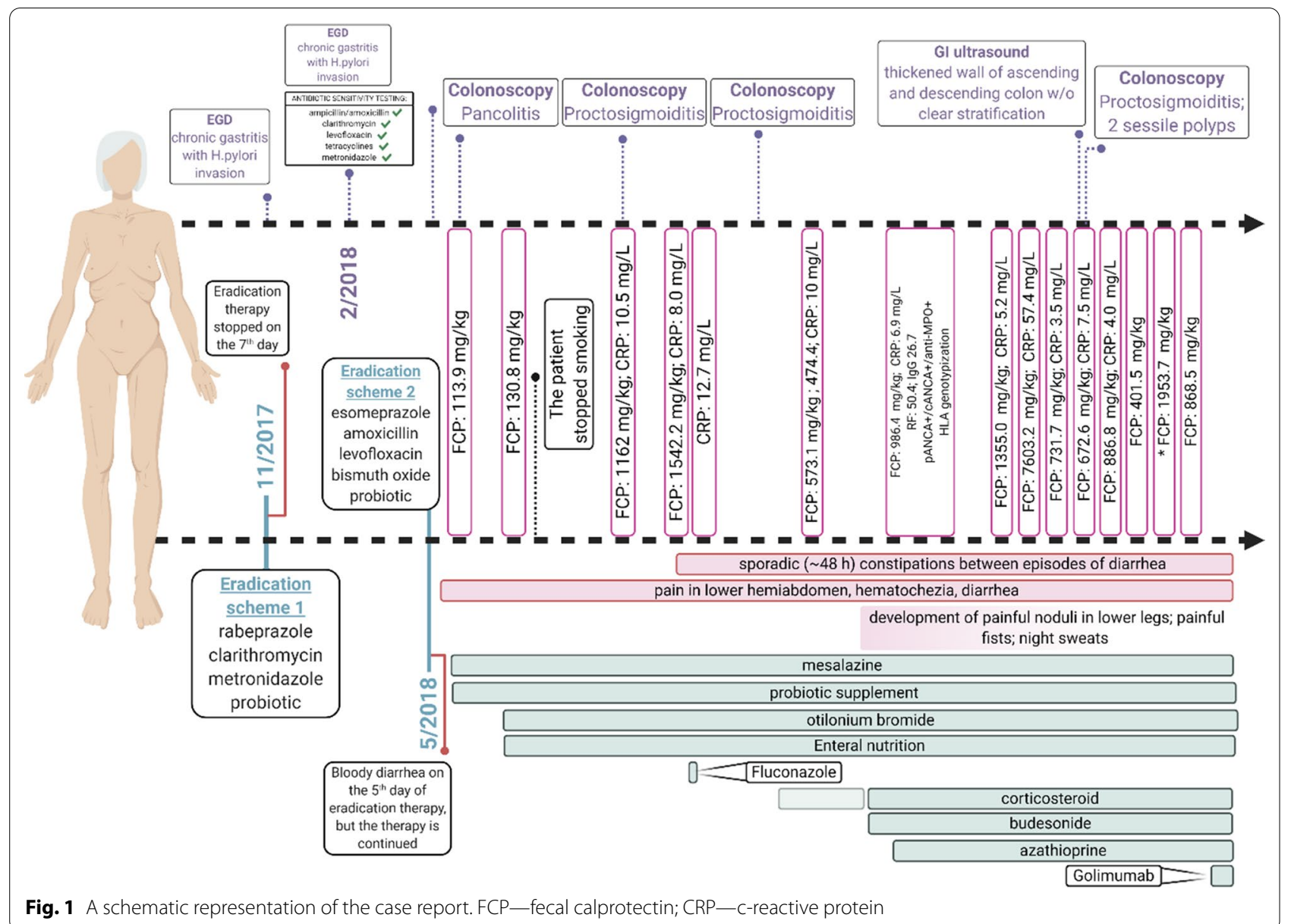

amoxicillin, levofloxacin, bismuth oxide, and a probiotic was proposed for eradication.

Five days in eradication, the patient developed bloody diarrhea but remained highly motivated to continue with the regimen despite the adverse drug reactions (ADRs). Upon successful completion of eradication, colonoscopy was done due to persistent bloody diarrhea. Edematous mucosa of the entire colon and internal hemorrhoids (grade I/II) were revealed and pathohistology was in concordance with pancolitis (Fig. 2C). C.difficile (Clostridioides difficile toxin A/B Enzyme-linked Fluorescent Assay VIDAS kit (bioMérieux, Marcy l'Etoile, France); detection limit: toxin A [7.73 ng/ml], toxin B [4.55 ng/ml]) and O\&P exam were negative. Fecal calprotectin (FCP) was $113.9 \mathrm{mg} / \mathrm{kg}$. The patient was prescribed a light dietary regimen, mesalazine (oral+enema). On the 10th day, a follow-up colonoscopy revealed persistent pancolitis and the patient reported diffuse pain in the lower hemiabdomen, hematochezia, and diarrhea. Successful eradication of $H$. pylori was confirmed with a stool antigen test. The patient was encouraged to remain adherent to the dietary regimen and therapy.
After 2 months, the patient reported a reduction in symptom severity in the period of therapeutic adherence, however, diarrhea persisted despite therapy. In a period of noncompliance, she noticed traces of blood in the stool that disappeared upon a continuation of the recommended therapy. Bacteriological, C.difficile, and stool O\&P remained unremarkable, and FCP was $130.8 \mathrm{mg} / \mathrm{kg}$. Otilonium bromide and meal replacement were added to the therapy. The patient stopped smoking.

After 4 months, symptom progression was reported: 3 watery stools/day with traces of blood that partially resolved with increased mesalazine. Esomeprazole was added to therapy. Bacteriological, C.difficile, O\&P, and H. pylori remained unremarkable. FCP increased to $1162 \mathrm{mg} / \mathrm{kg}$ and c-reactive protein (CRP) was $10.5 \mathrm{mg} / \mathrm{L}$. Colonoscopy revealed proctosigmoiditis, $1.5 \mathrm{~cm}$ flat cecal polyp, and several individual diverticula (Fig. 2D). Immunosuppressive therapy was recommended, but the patient declined due to fear of ADRs. Fluconazole was initiated as mycological stool testing revealed abundant Candida spp.. CRP was $8.0 \mathrm{mg} / \mathrm{L}$ and carcinoembryonic antigen was $6.2 \mathrm{ng} / \mathrm{ml}$. The patient reported watery stools 


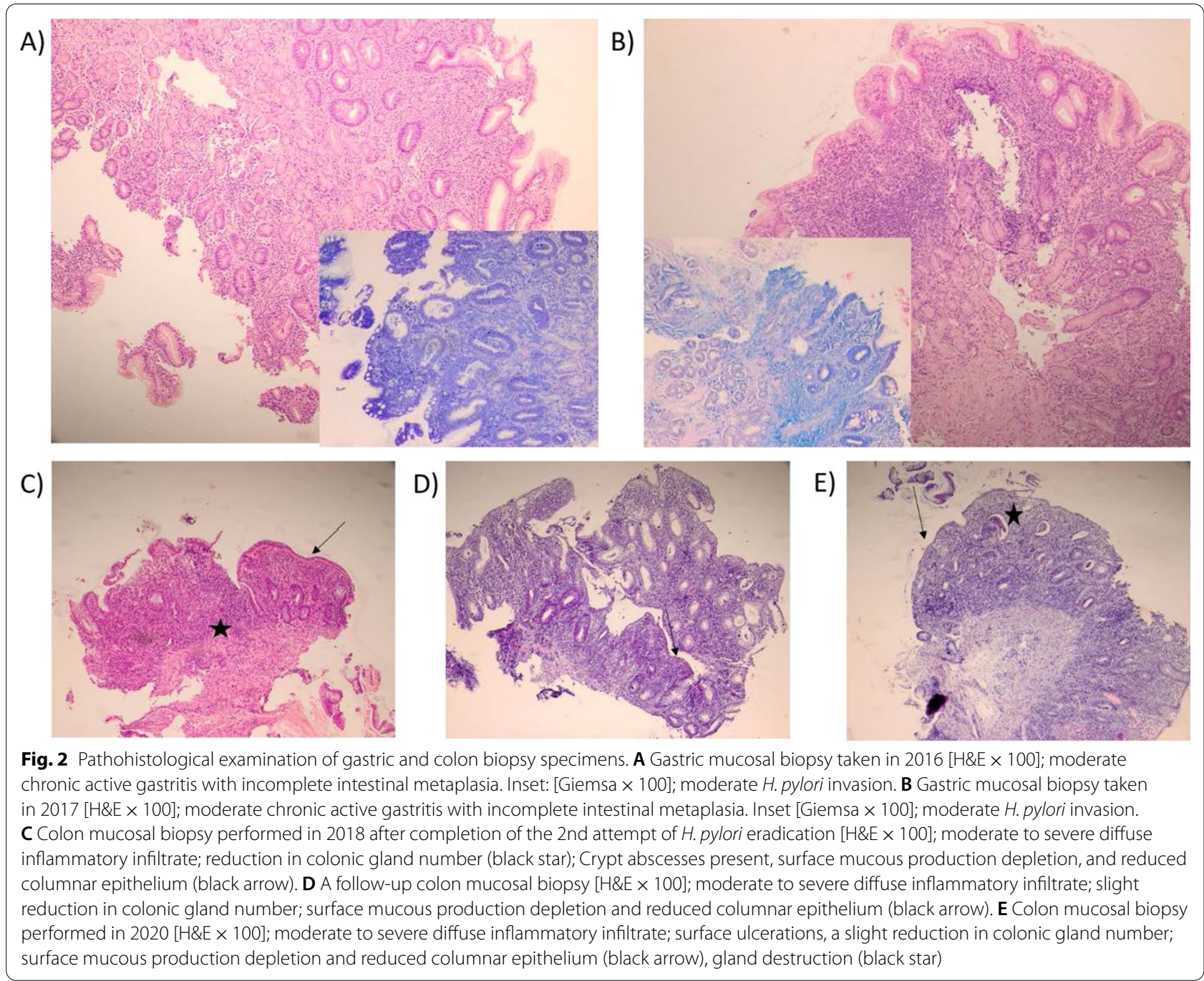

began to alternate with $\sim 48$ h-long periods of constipation. Pain in the lower hemiabdomen was persistent.

Two months later, a follow-up colonoscopy confirmed proctosigmoiditis (Fig. 2E). Corticosteroid treatment was recommended and a reduction in symptom severity was reported over the next two months although the patient admitted to variable compliance.

Three months later, the patient reported the development of painful shin nodules, pain in her wrists, and increased night sweats. The reported symptoms were considered a sign of enteropathic arthritis and methylprednisolone was initiated. Budesonide was added to therapy and celecoxib, denosumab, and vitamin D were initiated due to osteoporosis. Laboratory findings revealed FCP $986 \mathrm{mg} / \mathrm{kg}$; CRP 6,9 mg/l; rheumatoid factor $50.4 \mathrm{IU} / \mathrm{ml}$; IgG 26,7 g/l; pANCA (1:5120); cANCA (+); anti-MPO $103.9 \mathrm{IU} / \mathrm{ml}$; negative M.tuberculosis quantiferon test $(0.01 \mathrm{IU} / \mathrm{ml})$. HLA-typization indicated no relevant TPMT mutations and azathioprine was included in therapy.

Clinical improvement was observed after two months. Painful indurations regressed and the patient reported no pain in her wrists. There was 1-2 stools/day with sporadic traces of blood and mild diarrhea. The disease was now partially controlled with a combination of corticosteroid, mesalazine, and azathioprine, however, initiation of golimumab was recommended to avoid complications of steroid-dependent UC.

Golimumab therapy was initiated with a starting dose of $200 \mathrm{mg}$ (sc.) followed by additional $100 \mathrm{mg}$ sc. 2 weeks later, and $100 \mathrm{mg}$ sc. maintenance dose planned every month. So far the patient reported symptom improvement. Corticosteroid and azathioprine were successfully discontinued without a rebound. The disease is currently controlled with a combination of golimumab and mesalazine. 


\section{Discussion and conclusions}

We present a case of an elderly woman developing UC upon H. pylori eradication with a 3-year follow-up of the progression of the disease that eventually evolved into steroid-dependent UC complicated with enteropathic arthritis that was finally contained with golimumab. Initially, the patient's main concern was mild dyspepsia accompanied with EGD and PHD suggestive of chronic active gastritis with $H$. pylori invasion. Eradication was an obvious next step based on current guidelines [2]. Unfortunately, our patient suffered from ADRs and the therapy had to be stopped after 7 days. Eradication therapy ADRs are relatively common and expected [23], so a second eradication attempt was planned 2 months later after penicillin allergy and $H$. pylori antibiotic sensitivity testing to plan the optimal treatment strategy [24]. Unfortunately, bloody diarrhea developed on the 5th day, and colonoscopy revealed pancolitis that was initially considered either antibiotic-associated or hemorrhagic. Nevertheless, as bloody diarrhea persisted even after the therapy termination, the patient reported a relief following initiation of mesalazine, colonoscopy and PHD indicated UC, and other differential diagnoses were ruled out (e.g. C.difficile fecal toxin A and B, O\&P)-the patient was diagnosed with ulcerative proctosigmoiditis. Interestingly, a similar pattern of events was observed in a 63-year-old who developed bloody diarrhea upon $H$. pylori eradication therapy that was initially understood as antibiotic-associated colitis, but the condition didn't resolve on its own following treatment discontinuation [22]. Although both patients developed UC upon treatment with the H. pylori eradication therapy, several differences should be emphasized. In [22], eradication therapy-induced UC was transient and resolved within 3 months following 24 days of $750 \mathrm{mg} / \mathrm{kg} /$ day metronidazole [22]. Furthermore, although the UC-like signs and symptoms appeared in parallel with the $H$. pylori eradication regimen, a subsequent urea breath test indicated the infection was still present. As further information regarding $H$. pylori presence was not reported, the patient might have been $H$. pylori positive throughout transient UC. In contrast, $H$. pylori's absence was confirmed in our patient. Furthermore, UC severity and clinical course were less favorable for our patient. Fortunately, the disease is currently under control with golimumab, a reassuring finding considering the benefit of golimumab maintenance therapy was previously observed in patients who responded to golimumab induction $[25,26]$.

The presented case speaks in favor of the inverse association between $H$. pylori infection/exposure and prevalence/incidence of IBD proposed by numerous studies $[5,10-13,27-33]$. The majority of studies reporting an inverse relationship between $H$. pylori and IBD are ill-equipped to provide a mechanistic explanation for the observed effects. Nevertheless, recent evidence from preclinical experiments suggest chronic $H$. pylori infection may protect against IBD by inducing systemic immune tolerance and suppressing inflammatory responses [7]. For example, $H$. pylori infection reduces systemic inflammation and splenic $\mathrm{CD} 4^{+} \mathrm{T}$ cells and ameliorates colitis-associated histopathological changes in the dextran sulfate sodium-induced mouse model of colitis. Furthermore, in mice infected with Salmonella typhimurium, a co-infection with $H$. pylori increased mesenteric lymph node interleukin-10 and prevented the TH17 response [7]. In a recent comprehensive review of $H$. pyloriinduced immunomodulation activation of dendritic cell tolerogenic phenotype and immunosuppressive regulatory $\mathrm{T}$ cells were proposed as the most important mechanisms mediating potentially beneficial effects in IBD [7].

Interestingly, some studies reported no association between $H$. pylori and UC [34], and some even proposed it may act as a causative agent triggering the development of the disease. For example, Mansour et al. analyzed colonic biopsies from 30 patients newly diagnosed with $\mathrm{UC}$ and 30 controls and found $H$. pylori in $56.6 \%$ of cases and $20 \%$ of controls using polyclonal anti-H. pylori antibodies [35].

One possible explanation of the apparently paradoxical findings of the protective/harmful effects of $H$. pylori in IBD is that the differences in seroprevalence between IBD and the controls are a consequence of pharmacotherapy. For example, the protective role of 5-aminosalicylic acid on $H$. pylori infection has been proposed [36]. Similarly, some authors suggested other drugs such as metronidazole, corticosteroids, sulfasalazine or quinolones could also be responsible for the observed differences [7]. Nevertheless, several studies found no effect of IBD drugs on $H$. pylori seroprevalence [7].

\section{Strengths and limitations}

Strengths: (1) long-term follow-up; (2) defined early course of the disease (pancolitis evolving to proctosigmoiditis) as colonoscopy of the only other patient [22] reported 40 days after eradication suggested proctosigmoiditis; (3) differential diagnoses were ruled out (eg. bacteriological and O\&P stool examinations); (4) $H$. pylori eradication was confirmed; (5) biochemical indicators of disease severity were monitored and reported.

Limitations: We cannot rule-out that (1) our patient would develop UC regardless of the $H$. pylori eradication ( $20 \%$ of patients do after the age of 60 [37]); (2) UC onset was treatment and not $H$. pylori related. Although the risk of autoimmune diseases (including IBD) is increased in patients with peptic ulcer disease (PUD) who receive anti-H. pylori therapy, the same is true for PUD 
patients who didn't receive therapy and for patients who received antibiotics for urinary infection [38]. Antibiotics have been suggested both as risk factors and potential therapy for IBD. A recent systematic review on antibiotic exposure and risk of IBD suggested there might be an association [39], and a large population-based retrospective cohort indicated increased risk in subjects exposed to antianaerobic antibiotics [40]. On contrary, many randomized controlled trials reported antibiotics to be effective for induction of UC remission [41] and several meta-analyses suggest overall higher remission rates with antibiotics in comparison with placebo [42, 43]. The fact that the patient stopped smoking as well as that she had underlying thyroid disease should also be acknowledged in the context of UC as both are potential factors that could have affected the course of the disease. Smoking was found to be protective in UC, decreasing the need for colectomy in some patients possibly due to the effect on cellular and humoral immune responses and mucus production [44]. Furthermore, it has been reported that autoimmune disorders are more frequent in patients with IBD indicating possibly overlapping pathophysiological processes and/or shared risk factors [45]. We cannot rule out that both factors affected the course of the disease. Nevertheless, smoking cessation could not have caused $\mathrm{UC}$ as the patient stopped smoking well after the disease developed. On the other hand, genetic susceptibility to autoimmune diseases could have contributed to the predisposition for subsequent development of UC.

\section{The primary "take-away"}

Taking into account a consensus that all infected individuals should be treated with $H$. pylori eradication therapy, it is to be expected that rare cases of eradication-induced UC might become more prevalent. In our patient, an unfortunate combination of environmental and genetic risk factors (predisposition to autoimmune disorders, e.g. Hashimoto's thyroiditis), complicated with $H$. pylori eradication-induced dysbiosis triggered pathophysiological events leading to the development of a serious case of UC. In the future, a better understanding of the molecular mechanisms responsible for the disease development, factors influencing the protective and/or harmful effects of $H$. pylori (e.g. recent report indicated protective effects of $H$. pylori in IBD are mediated by the cytotoxin-associated gene A positive strain [46], and there is some evidence of mediation by the protective cross-reactivity to IBD aggravating C.jejuni [5]), and personalized approach to the $H$. pylori eradication might help inform a new set of guidelines based on maximizing the beneficial effect of eradication, while minimizing the risk of potential side effects of eradication treatment.

\section{Abbreviations}

H. pylori: Helicobacter pylori; IBD: Inflammatory bowel disease; UC: Ulcerative colitis; CD: Crohn's disease; CT: Computed tomograpy; EGD: Esophagogastroduodenoscopy; ADRs: Adverse drug reactions; FCP: Fecal calprotectin; CRP: C-reactive protein; PUD: Peptic ulcer disease.

\section{Acknowledgements}

We wish to express our gratitude to the patient who grant us a permission to publish this case.

\section{Authors' contributions \\ $J \mathrm{H}$ wrote the manuscript. MN, MZ, DB, IB, IP, DH and NJ were directly involved with the case. MN, MZ, DB, IB, IP, DH, NJ, DP and DV commented on the manu- script and provided valuable feedback. MN supervised the project. All authors read and approved the manuscript.}

\section{Funding}

None.

\section{Availability of data and materials}

Data will be provided upon reasonable request and in concordance with maximal protection of the patient privacy.

\section{Declarations}

Ethics approval and consent to participate

Informed consent has been obtained from the patient.

\section{Consent for publication}

Written informed consent was obtained from the patient for publication of this case report and any accompanying images. A copy of the written consent is available for review by the Editor of this journal.

\section{Competing interests}

The authors have no conflict of interest to disclose.

\section{Author details}

${ }^{1}$ Department of Pharmacology, University of Zagreb School of Medicine, Zagreb, Croatia. ${ }^{2}$ Gastroenterology and Hepatology Unit, University Hospital Centre "Sestre Milosrdnice", Vinogradska 29, 10000 Zagreb, Croatia. ${ }^{3}$ University of Zagreb School of Dental Medicine, Zagreb, Croatia. ${ }^{4}$ University of Zagreb School of Medicine, Zagreb, Croatia. ${ }^{5}$ Department of Pathology, University Hospital Centre "Sestre Milosrdnice", Zagreb, Croatia. ${ }^{6}$ Department of Periodontology, University of Zagreb School of Dental Medicine, Zagreb, Croatia.

Received: 4 April 2021 Accepted: 6 July 2021

Published online: 31 July 2021

\section{References}

1. Khoder G, Muhammad JS, Mahmoud I, Soliman SSM, Burucoa C. Prevalence of Helicobacter pylori and its associated factors among healthy asymptomatic residents in the United Arab Emirates. Pathogens. 2019;8:44.

2. Malfertheiner P, Megraud F, O'Morain CA, Gisbert JP, Kuipers EJ, Axon AT, et al. Management of Helicobacter pylori infection - the Maastricht V/ Florence consensus report. Gut. 2017;66:6-30.

3. Sugano K, Tack J, Kuipers EJ, Graham DY, El-Omar EM, Miura S, et al. Kyoto global consensus report on Helicobacter pylori gastritis. Gut. 2015;64:1353-67.

4. Zuo ZT, Ma Y, Sun Y, Bai CQ, Ling CH, Yuan FL. The protective effects of Helicobacter pylori Infection on allergic asthma. IAA. 2021;182:53-64.

5. Castaño-Rodríguez N, Kaakoush NO, Lee WS, Mitchell HM. Dual role of Helicobacter and Campylobacter species in IBD: a systematic review and meta-analysis. Gut. 2017;66:235-49.

6. Guan Q. A comprehensive review and update on the pathogenesis of inflammatory bowel disease. J Immunol Res. 2019;2019:1-16. 
7. Yu Y, Zhu S, Li P, Min L, Zhang S. Helicobacter pylori infection and inflammatory bowel disease: a crosstalk between upper and lower digestive tract. Cell Death Dis. 2018;9:1-12.

8. Abdel Razek AA, Abu Zeid MM, Bilal M, Abdel Wahab NM. Virtual CT colonoscopy versus conventional colonoscopy: a prospective study. Hepatogastroenterology. 2005;52:1698-702.

9. Abd-El Khalek Abd-ALRazek A, Fahmy DM. Diagnostic value of diffusionweighted imaging and apparent diffusion coefficient in assessment of the activity of Crohn disease: 1.5 or 3 T. J Comput Assist Tomogr. 2018:42:688-96

10. Wu X-W, Ji H-Z, Yang M-F, Wu L, Wang F-Y. Helicobacter pylori infection and inflammatory bowel disease in Asians: a meta-analysis. World J Gastroenterol. 2015;21:4750-6.

11. Song MJ, Park DI, Hwang SJ, Kim ER, Kim YH, Jang BI, et al. The prevalence of Helicobacter pylori infection in Korean patients with inflammatory bowel disease, a multicenter study. Korean J Gastroenterol. 2009;53:341-7.

12. Sayar R, Shokri Shirvani J, Hajian-Tilaki K, Vosough Z, Ranaei M. The negative association between inflammatory bowel disease and Helicobacter pylori seropositivity. Caspian J Intern Med. 2019;10:217-22.

13. Jin X, Chen Y, Chen S, Xiang Z. Association between Helicobacter pylori infection and ulcerative colitis-a case control study from China. Int J Med Sci. 2013;10:1479-84.

14. Gravina AG, Priadko K, Ciamarra P, Granata L, Facchiano A, Miranda A, et al. Extra-gastric manifestations of Helicobacter pylori infection. J Clin Med. 2020;9:3887.

15. Gravina A, Prevete N, Tuccillo C, De Musis C, Romano L, Federico A, et al. Peptide $\mathrm{Hp}(2-20)$ accelerates healing of TNBS-induced colitis in the rat. $U$ Eur Gastroenterol J. 2018;6:1428-36. https://doi.org/10.1177/2050640618 793564.

16. Khan I, Ullah N, Zha L, Bai Y, Khan A, Zhao T, et al. Alteration of gut microbiota in inflammatory bowel disease (IBD): cause or consequence? IBD treatment targeting the gut microbiome. Pathogens. 2019:8:126.

17. Heimesaat MM, Fischer A, Plickert R, Wiedemann T, Loddenkemper C, Göbel UB, et al. Helicobacter pylori induced gastric immunopathology is associated with distinct microbiota changes in the large intestines of long-term infected Mongolian gerbils. PLoS ONE. 2014;9:e100362.

18. Burisch J, Jess T. Does eradication of Helicobacter pylori cause inflammatory bowel disease? Clin Gastroenterol Hepatol. 2019;17:1940-1.

19. Jovanovic IR, Milosavjevic TN, Jankovic GP, Micev MM, Dugalic PD, Saranovic D, et al. Clinical onset of the Crohn's disease after eradication therapy of Helicobacter pylori infection does Helicobacter pylori infection interact with natural history of inflammatory bowel diseases? Med Sci Monit. 2001;7:137-41.

20. Tursi A. Onset of Crohn's disease after Helicobacter pylori eradication. Inflamm Bowel Dis. 2006;12:1008-9. https://doi.org/10.1097/01.mib. 0000235100.09231.d7.

21. Nagami H, Otani J, Ueda K. A clinical case of total ulcerative colitis which was rapidly progressed after Helicobacter pylori removal therapy. Shokakika (Gastroenterology). 2008;47(2):205-10.

22. Chiba M, Tsuji T, Takahashi K, Komatsu M, Sugawara T, Ono I. Onset of ulcerative colitis after Helicobacter pylori eradication therapy: a case report. Perm J. 2016;20:e115-118.

23. Gebeyehu E, Nigatu D, Engidawork E. Self-reported adverse drug effects and associated factors among $\mathrm{H}$. pylori infected patients on standard triple therapy: prospective follow up study. PLoS ONE. 2019;14:e0225585.

24. Smith SM, O'Morain C, McNamara D. Antimicrobial susceptibility testing for Helicobacter pylori in times of increasing antibiotic resistance. World Gastroenterol. 2014;20:9912-21.

25. Sandborn WJ, Feagan BG, Marano C, Zhang H, Strauss R, Johanns J, et al. Subcutaneous golimumab maintains clinical response in patients with moderate-to-severe ulcerative colitis. Gastroenterology. 2014;146:96-109. e1.

26. Kokkinidis DG, Bosdelekidou EE, lliopoulou SM, Tassos AG, Texakalidis PT, Economopoulos KP, et al. Emerging treatments for ulcerative colitis: a systematic review. Scand J Gastroenterol. 2017;52:923-31.
27. Halme L, Rautelin H, Leidenius M, Kosunen TU. Inverse correlation between Helicobacter pylori infection and inflammatory bowel disease. J Clin Pathol. 1996;49:65-7.

28. Sukerek HH, Thomas RL, Tolia VK. Incidence of Helicobacter pylori infection in pediatric inflammatory bowel disease patients. Am J Gastroenterol. 2001;96:S310.

29. Prónai L, Schandl L, Orosz Z, Magyar P, Tulassay Z. Lower prevalence of Helicobacter pylori infection in patients with inflammatory bowel disease but not with chronic obstructive pulmonary disease - antibiotic use in the history does not play a significant role. Helicobacter. 2004;9:278-83.

30. Roka K, Roubani A, Stefanaki K, Panayotou I, Roma E, Chouliaras G. The prevalence of Helicobacter pylori gastritis in newly diagnosed children with inflammatory bowel disease. Helicobacter. 2014;19:400-5.

31. Zhang S, Zhong B, Chao K, Xiao Y, Cui Y, Gao X, et al. Role of Helicobacter species in Chinese patients with inflammatory bowel diseasev. J Clin Microbiol. 2011;49:1987-9.

32. Sonnenberg A, Genta RM. Low prevalence of Helicobacter pylori infection among patients with inflammatory bowel disease. Aliment Pharmacol Ther. 2012;35:469-76.

33. Luther J, Dave M, Higgins PDR, Kao JY. Association between Helicobacter pylori infection and inflammatory bowel disease: a meta-analysis and systematic review of the literature. Inflamm Bowel Dis. 2010;16:1077-84.

34. Oliveira AG, Sanna M, das GP, Rocha GA, Rocha AMC, Santos A, Dani $\mathrm{R}$, et al. Helicobacter species in the intestinal mucosa of patients with ulcerative colitis. J Clin Microbiol. 2004;42:384-6.

35. Mansour L, El-Kalla F, Kobtan A, Abd-Elsalam S, Yousef M, Soliman S, et al. Helicobacter pylori may be an initiating factor in newly diagnosed ulcerative colitis patients: a pilot study. World J Clin Cases. 2018;6:641-9.

36. Piodi LP, Bardella M, Rocchia C, Cesana BM, Baldassarri A, Quatrini M. Possible protective effect of 5-aminosalicylic acid on Helicobacter pylori infection in patients with inflammatory bowel disease. J Clin Gastroenterol. 2003;36:22-5.

37. Ananthakrishnan AN, Donaldson T, Lasch K, Yajnik V. Management of inflammatory bowel disease in the elderly patient: challenges and opportunities. Inflamm Bowel Dis. 2017;23:882-93.

38. Lin K-D, Chiu G-F, Waljee AK, Owyang SY, El-Zaatari M, Bishu S, et al. Effects of anti-Helicobacter pylori therapy on incidence of autoimmune diseases, including inflammatory bowel diseases. Clin Gastroenterol Hepatol. 2019;17:1991-9.

39. Theochari NA, Stefanopoulos A, Mylonas KS, Economopoulos KP. Antibiotics exposure and risk of inflammatory bowel disease: a systematic review. Scand I Gastroenterol. 2018:53:1-7.

40. Kronman MP, Zaoutis TE, Haynes K, Feng R, Coffin SE. Antibiotic exposure and IBD development among children: a population-based cohort study. Pediatrics. 2012;130:e794-803.

41. Ledder O. Antibiotics in inflammatory bowel diseases: do we know what we're doing? Transl Pediatr. 2019;8:42-55.

42. Rahimi R, Nikfar S, Rezaie A, Abdollahi M. A meta-analysis of antibiotic therapy for active ulcerative colitis. Dig Dis Sci. 2007;52:2920-5.

43. Khan KJ, Ullman TA, Ford AC, Abreu MT, Abadir A, Abadir A, et al. Antibiotic therapy in inflammatory bowel disease: a systematic review and meta-analysis. Am J Gastroenterol. 2011;106:661-73.

44. Lakatos PL. Environmental factors affecting inflammatory bowel disease: have we made progress? Dig Dis. 2009;27:215-25.

45. Halling ML, Kjeldsen J, Knudsen T, Nielsen J, Hansen LK. Patients with inflammatory bowel disease have increased risk of autoimmune and inflammatory diseases. World J Gastroenterol. 2017;23:6137-46.

46. Lord AR, Simms LA, Hanigan K, Sullivan R, Hobson P, Radford-Smith GL. Protective effects of Helicobacter pylori for IBD are related to the cagApositive strain. Gut. 2018:67:393-4.

\section{Publisher's Note}

Springer Nature remains neutral with regard to jurisdictional claims in published maps and institutional affiliations. 